\title{
Solução Numérica de Equações Diferenciais Rígidas
}

\author{
Willian C. Lesinhovski* ${ }^{*} \quad$ Rudimar L. Nós \\ Departamento Acadêmico de Matemática, UTFPR \\ 80230-901, Curitiba, PR \\ E-mail: wlesin@yahoo.com.br, rudimarnos@utfpr.edu.br
}

\section{$\underline{\text { RESUMO }}$}

Este trabalho é um estudo inicial sobre a solução numérica de equações diferenciais ordinárias rígidas. Investigou-se, dentre alguns métodos numéricos de passo simples e de passo múltiplo, explícitos e implícitos, aqueles que fornecem as melhores aproximações para a solução de uma equação diferencial ordinária rígida.

Uma equação diferencial é dita rígida quando a sua solução exata tem um termo da forma $e^{-c t}$, onde $c>>0$ e $t>0$. Este termo, que decai rapidamente para zero à medida que $t$ aumenta, é a parte transiente da solução. A rigidez é caracterizada pela existência de duas ou mais escalas distintas para a variável independente $[1,2,3]$. Problemas de valor inicial com equações rígidas são relativamente comuns, particularmente no estudo de vibrações, reações químicas e circuitos elétricos.

Palavras-chave: Equações diferenciais ordinárias rígidas, métodos de passo simples, métodos de passo múltiplo, métodos explícitos, métodos implícitos.

\section{Problema de Cauchy}

Selecionou-se o seguinte problema de valor inicial (p.v.i.) rígido [1]:

$$
\begin{aligned}
\frac{d}{d t} y(t) & =\frac{5}{2} t^{2}+\frac{1}{2} t-10 y(t), \quad t \in[0,5] \\
y(0) & =4 \\
f_{n} & =f\left(t_{n}, y_{n}\right)=\frac{5}{2} t_{n}^{2}+\frac{1}{2} t_{n}-10 y_{n} \\
y(t) & =4 e^{-10 t}+\frac{1}{4} t^{2}
\end{aligned}
$$

Para solucionar numericamente o p.v.i. (1), cria-se uma malha de $n+1$ pontos do domínio da função $y(t), t_{0}<t_{1}<\ldots<t_{n}$, e em cada ponto $t_{k}$ dessa malha usa-se o valor aproximado de $y(t)$ em um ou mais pontos para calcular o valor aproximado de $y(t)$ em $t_{k}$, denotado por $y_{k}$. A diferença $t_{k}-t_{k-1}=h, k=1,2, \ldots, n$, é o passo de integração, o qual, neste trabalho, é constante.

\section{Métodos numéricos}

Método de Euler

$$
y_{n+1}=y_{n}+h f_{n}, \quad n=0,1,2, \ldots
$$

*Bolsista de Iniciação Científica PIBIC/UTFPR 
Método de Euler Implícito

$$
y_{n+1}=y_{n}+h f_{n+1}, \quad n=0,1,2, \ldots
$$

Método de Euler Aprimorado

$$
y_{n+1}=y_{n}+\frac{h}{2}\left(f_{n}+f_{n+1}\right), \quad n=0,1,2, \ldots
$$

Método de Runge-Kutta 44

$$
\begin{aligned}
y_{n+1} & =y_{n}+\frac{h}{6}\left(k_{n 1}+2 k_{n 2}+2 k n 3+k_{n 4}\right), \quad n=0,1,2, \ldots \\
k_{n 1} & =f\left(t_{n}, y_{n}\right), k_{n 2}=f\left(t_{n}+\frac{1}{2} h, y_{n}+\frac{1}{2} h k_{n 1}\right) \\
k_{n 3} & =f\left(t_{n}+\frac{1}{2} h, y_{n}+\frac{1}{2} h k_{n 2}\right), k_{n 4}=f\left(t_{n}+h, y_{n}+h k_{n 3}\right)
\end{aligned}
$$

Método de Adams-Bashforth

$$
y_{n+1}=y_{n}+\frac{h}{24}\left(55 f_{n}-59 f_{n-1}+37 f_{n-2}-9 f_{n-3}\right), \quad n=0,1,2, \ldots
$$

Método de Adams-Moulton

$$
y_{n+1}=y_{n}+\frac{h}{24}\left(9 f_{n+1}+19 f_{n}-5 f_{n-1}+f_{n-2}\right), \quad n=0,1,2, \ldots
$$

Os métodos (5),(6) e (9) são implícitos, pois torna-se necessário solucionar uma equação algébrica para determinar $y_{n+1}$; os demais métodos são explícitos. Além disso, os métodos (8) e (9) são ditos de passo múltiplo, pois dependem de mais de um ponto anterior para calcular $y_{n+1}$, ao contrário dos métodos (4)-(7), de passo simples ou passo único, que dependem do ponto imediatamente anterior. Nos métodos (8) e (9), empregou-se o método (7) para calcular $y_{1}, y_{2}$ e $y_{3}$. Implementou-se os métodos (4)-(9) em linguagem C [4] e utilizou-se o Matlab para visualizar os resultados (Figura 1).

\section{Resultados numéricos}

A Tabela (1) e a Figura (1) mostram a norma do máximo do erro absoluto cometido ( $\left.\|e\|_{\infty}\right)$ e o tempo médio de processamento $\left(t_{C P U}\right)$ dos métodos (4)-(9). Como a função que mede o tempo de processamento em $\mathrm{C}$ é imprecisa, para cada método e passo de integração repetiu-se o processo 1000 vezes e considerou-se a média aritmética como tempo médio.

\section{Conclusões}

Através da análise dos resultados obtidos, conclui-se que os métodos implícitos fornecem, em geral, aproximações melhores do que os explícitos para passos de integração maiores, o que evidencia maior região de estabilidade $[1,2,3]$.

\section{Referências}

[1] W.E. Boyce; R.C. Diprima, "Equações diferenciais elementares e problemas de valores de contorno", 9 ed., LTC, Rio de Janeiro, 2006. 
Tabela 1: Norma do máximo do erro absoluto e tempo médio de processamento na solução numérica do p.v.i. (1) empregando-se os métodos (4)-(9) com diferentes passos de integração $h$.

\begin{tabular}{|c|c|c|c|c|}
\hline Método & e & $t_{C P U}$ & e & $t_{C P U}$ \\
\hline & \multicolumn{2}{|c|}{$\mathrm{h}=\mathbf{0 , 5}$} & \multicolumn{2}{|c|}{$\mathrm{h}=\mathbf{0 , 2 5}$} \\
\hline Euler & $4,207411.10^{6}$ & $2,6670.10^{-3}$ & $1,332180.10^{4}$ & $4,7740.10^{-3}$ \\
\hline Euler Implícito & $6,501315.10^{-1}$ & $2,4650.10^{-3}$ & $8,189814.10^{-1}$ & $4,7110.10^{-3}$ \\
\hline Euler Aprimorado & $1,741238.10^{0}$ & $2,5120.10^{-3}$ & $7,727844.10^{-1}$ & $4,7740.10^{-3}$ \\
\hline Runge-Kutta 44 & $9,403616.10^{11}$ & $2,9010.10^{-3}$ & $2,270496.10^{0}$ & $4,6960.10^{-3}$ \\
\hline Adams-Bashforth & $2,412668.10^{11}$ & $2,9950.10^{-3}$ & $2,788496.10^{12}$ & $5,8810.10^{-3}$ \\
\hline \multirow[t]{2}{*}{ Adams-Moulton } & $6,064083.10^{3}$ & $2,6320.10^{-3}$ & $2,270496.10^{0}$ & $4,8900.10^{-3}$ \\
\hline & \multicolumn{2}{|c|}{$\mathrm{h}=0,125$} & \multicolumn{2}{|c|}{$\mathrm{h}=0,0625$} \\
\hline Euler & $2,149925.10^{0}$ & $8,7510.10^{-3}$ & $6,420223.10^{-1}$ & $1,7254.10^{-2}$ \\
\hline Euler Implícito & $6,334947.10^{-1}$ & $9,1570.10^{-3}$ & $3,697445.10^{-1}$ & $1,8111.10^{-2}$ \\
\hline Euler Aprimorado & $2,229423.10^{-1}$ & $9,1890.10^{-3}$ & $4,851352.10^{-2}$ & $1,8096.10^{-2}$ \\
\hline Runge-Kutta 44 & $8,395747.10^{-2}$ & $9,2360.10^{-3}$ & $3.087647 .10^{-3}$ & $1,8111.10^{-2}$ \\
\hline Adams-Bashforth & $1,002599.10^{17}$ & $1,2090.10^{-2}$ & $7,483143.10^{15}$ & $2,2761.10^{-2}$ \\
\hline \multirow[t]{2}{*}{ Adams-Moulton } & $8,395747.10^{-2}$ & $9,3570.10^{-3}$ & $3,087647.10^{-3}$ & $1,8617.10^{-2}$ \\
\hline & \multicolumn{2}{|c|}{$\mathrm{h}=0,03125$} & \multicolumn{2}{|c|}{$\mathrm{h}=0,015625$} \\
\hline Euler & $2,671452.10^{-1}$ & $3,4429.10^{-2}$ & $1,234184.10^{-1}$ & $6,8312.10^{-2}$ \\
\hline Euler Implícito & $2,031526.10^{-1}$ & $3,6020.10^{-2}$ & $1,081811.10^{-1}$ & $7,1916.10^{-2}$ \\
\hline Euler Aprimorado & $1,208219.10^{-2}$ & $3,5958.10^{-2}$ & $2,995835.10^{-3}$ & $7,1963.10^{-2}$ \\
\hline Runge-Kutta 44 & $1,519257.10^{-4}$ & $3,6083.10^{-2}$ & $8,331014.10^{-6}$ & $7,2072.10^{-2}$ \\
\hline Adams-Bashforth & $3,199923.10^{-2}$ & $3,6894.10^{-2}$ & $2,472590.10^{-4}$ & $7,3320.10^{-2}$ \\
\hline Adams-Moulton & $2,105729.10^{-4}$ & $3,6809.10^{-2}$ & $1,741384.10^{-5}$ & $7,3229.10^{-2}$ \\
\hline
\end{tabular}

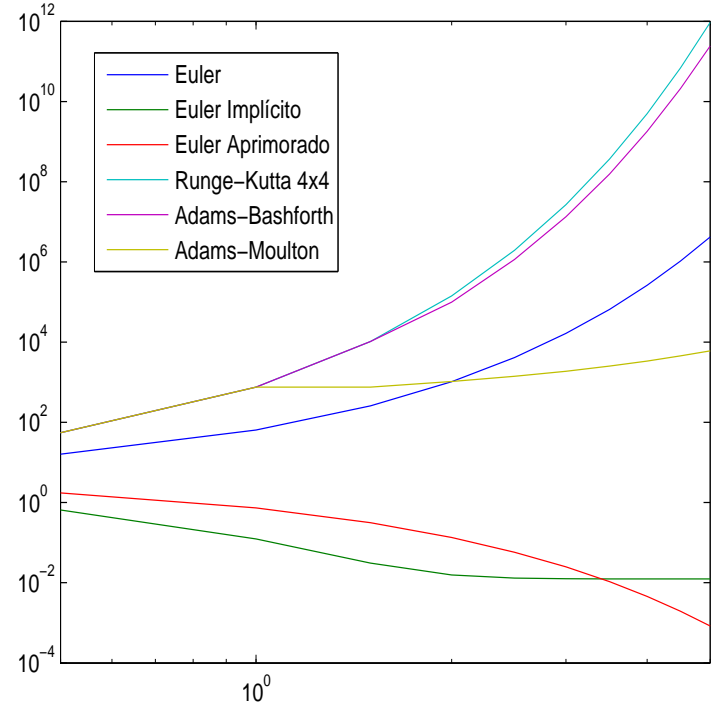

(a)

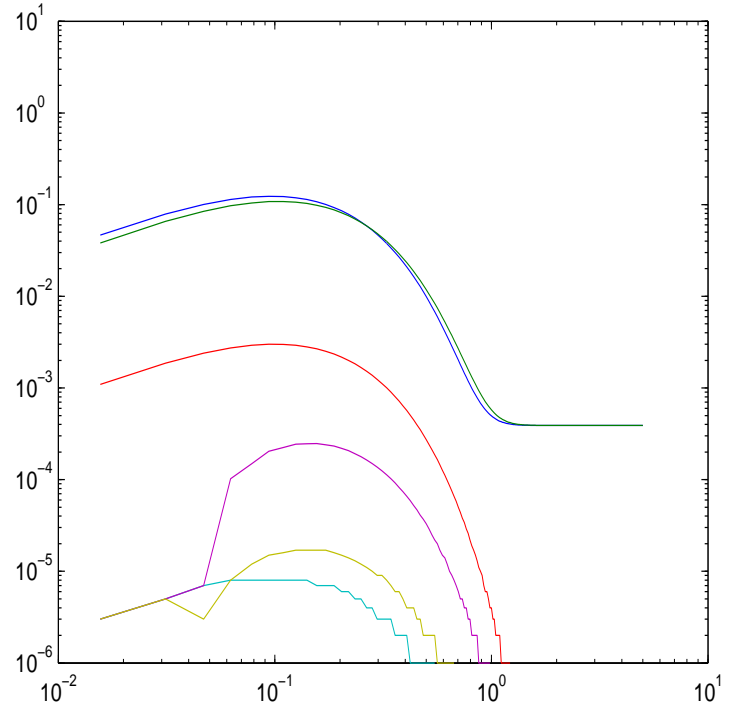

(b)

Figura 1: Norma do máximo do erro absoluto cometido na solução numérica do p.v.i. (1) empregando-se os métodos (4)-(9) com os seguintes passos de integração: (a) 0,5; (b) 0,015625.

[2] R.L. Burden; J.D. Faires, "Numerical analysis", 9th ed., Brooks/Cole, Boston, 2011.

[3] A.M. Roma, R.L. Nós, "Tratamento numérico de equações diferenciais - Notas de aula", IME-USP, São Paulo, 2012.

[4] H. Schildt, "C Completo e total", Makron Books, São Paulo, 1996. 